\title{
SIMULACIÓN DINÁMICA DE UNA COLUMNA DE DESTILACIÓN DE BIOETANOL EN RÉGIMEN BATCH
}

\section{DYNAMIC SIMULATION OF A DISTILLATION COLUMN OF BIOETHANOL IN BATCH REGIME}

\author{
Eliana Jara Morante ${ }^{1}$, Emerson Collado Dominguez ${ }^{2}$, \\ Mario De la Cruz Azabache ${ }^{3}$, Magali Vivas Cuellar' ${ }^{4}$. \\ RESUMEN
}

\begin{abstract}
La destilación batch es un proceso bastante empleado en numerosos procesos industriales a mediana y pequeña escala, por lo tanto de gran importancia comercial. En el contexto de la simulación de procesos, presenta retos ya que el sistema presenta mucha complejidad, especialmente en la etapa de startup donde existe variación de muchas variables simultáneamente. El presente trabajo simula el proceso de destilación de la planta piloto que se encuentra instalada en el Laboratorio de Operaciones Unitarias de la FIQT de la UNI. Para ello se hace uso del software simulación comercial ASPEN Batch Distillation V7.1. Los resultados nos sirven para establecer un protocolo de operación que nos permite obtener una alta pureza de bioetanol y mejorar el proceso.
\end{abstract}

Palabras clave.- Destilación batch, Bioetanol, Simulación.

\begin{abstract}
Batch distillation is a process used in many industrial processes, from small to big scale, therefore it has a great commercial interest. In the process simulation context it presents challenges due to the system complexity, especially in the startup stage because there are many variables changing simultaneously. This work simulates the distillation process of a bench plant installed in the Unit Operations Laboratory of the FIQT of the UNI. Commercial simulation software ASPEN Batch Distillation V7.1 is used. The results are useful to establish a protocol of operation that allows us to obtain high bioethanol purity and the improvement of the process.
\end{abstract}

Keywords.- Batch distillation, Bioethanol, Simulation.

\section{INTRODUCCIÓN}

La destilación batch es un método de separación bastante conocido. Desde el punto de vista de la simulación, es un proceso que ofrece bastantes retos, especialmente por la dificultad que representa simular la puesta en marcha (startup) hasta llevarlo al estado denominado seudoestacionario para obtener un producto de la calidad deseada. La complejidad del proceso para simularlo radica en que la puesta en marcha involucra el cambio drástico de varias variables simultáneamente. Muchos modelos se han generado para simular este proceso [1-6], y muchos investigadores coinciden en que para optimizarlo se requiere disminuir el tiempo en que el sistema alcanza el estado seudo-estacionario [3].

\footnotetext{
${ }^{1}$ Dra. Facultad de Ingeniería Química y Textil, Universidad Nacional de Ingeniería., ${ }^{2}$ Ing. Facultad de Ingeniería Química y Textil, Universidad Nacional de Ingeniería., ${ }^{3}$ Ing. Facultad de Ingeniería Química y Textil, Universidad Nacional de Ingeniería., ${ }^{4}$ Ing. Facultad de Ingeniería Química y Textil, Universidad Nacional de Ingeniería.
} 
En estos modelos la columna de destilación se describe modelando plato a plato a través de sus propiedades físicas y la geometría real del sistema, especialmente parámetros de los platos o empaque. Los modelos matemáticos que se han desarrollado se basan en balance de materia y energía con las ecuaciones de equilibrio generando sistemas de ecuaciones algebraicas y diferenciales de alto orden, en muchos casos estos modelos han demostrado ser rápidos y confiables. Desde el punto de vista industrial se debe disminuir el tiempo de solución sin dejar de alcanzar una solución confiable.

El equipo de destilación batch que se va a simular en este estudio se encuentra instalado en el laboratorio de operaciones unitarias (LOU) de la Facultad de Ingeniería Química y textil de la Universidad Nacional de Ingeniería (UNI). Se emplea para separar el etanol que se produce en la fermentación del jugo de caña de azúcar llevada a cabo en el bioreactor de la misma planta.

El trabajo presenta la simulación de la columna de destilación en régimen batch, con el fin de identificar las variables que afectan al proceso y al mismo tiempo evaluar la manera como se ha operado la destilación experimentalmente. Para ello ha usado el paquete de simulación comercial de ASPEN Batch Distillation V7.1. La simulación representa el proceso desde el momento de la carga, calentamiento y llegada al estado seudoestacionario para proceder a la obtención del destilado. En la simulación se ha logrado capturar la dinámica del proceso, lo que se comprueba con los resultados experimentales. Los resultados satisfactorios obtenidos nos permitirán implementar un mejor control y mejorar la producción y protocolo de trabajo.

\section{DESCRIPCIÓN DEL PROCESO}

La planta de separación de etanol-agua consta de una columna de destilación un condensador y un tanque de reflujo operando en régimen batch. Las características físicas de la planta se resumen en la Tabla 1.

La planta está diseñada para tratar una carga fresca máxima de 30 litros de mezcla por batch. La mezcla a destilar proviene de la fermentación de jugo de caña que se produce en el bioreactor.
Tabla 1 Características generales de la planta de destilación.

\begin{tabular}{|c|c|}
\hline $\begin{array}{l}\text { Columna } \\
\text { Nro. de etapas }\end{array}$ & 10 \\
\hline Rehervidor & Elíptico vertical \\
\hline Diámetro [cm] & 36 \\
\hline Altura [cm] & 33 \\
\hline Diámetro [cm] & 36 \\
\hline Altura [cm] & 33 \\
\hline Volumen $\left[\mathrm{cm}^{3}\right]$ & 45804 \\
\hline Área con liq. $\left[\mathrm{cm}^{2}\right]$ & 2645.92 \\
\hline Carga de vapor $[\mathrm{kW}]$ & 1.3 \\
\hline Presión vapor [psia] & $5-1$ \\
\hline Condensador & Elíptico horizontal \\
\hline Diámetro [cm] & 10.2 \\
\hline Longitud [cm] & 47 \\
\hline Área $\left[\mathrm{cm}^{2}\right]$ & 1632.65 \\
\hline Tanque & Elíptico horizontal \\
\hline Diámetro [cm] & 20.9 \\
\hline Longitud [cm] & 35 \\
\hline
\end{tabular}

El sistema de calentamiento del rehervidor es vía una chaqueta de calentamiento usando vapor saturado. El rehervidor tiene conectado un medidor de presión para medir la presión de vapor en la chaqueta de calentamiento y dos termopares para medir la temperatura de la carga y del vapor.

La columna de separación está hecha de acero de $1.65 \mathrm{~m}$ de longitud y está completamente aislada, minimizando de esta manera las pérdidas de calor con los alrededores. Se han conectados dos termopares dispuestos en el primer plato y el tope de la columna. El flujo del agua de enfriamiento y del condensado de vapor se mide periódicamente durante la operación conjuntamente con las temperaturas. El reflujo es controlado manualmente y la composición del destilado se determina in situ mediante un picnómetro (grados Gay Lussac).

\section{RESULTADOS EXPERIMENTALES}

\section{Procedimiento experimental}

En una corrida se carga la mezcla en el rehervidor y se sella adecuadamente, luego se procede a abrir las válvulas de agua de enfriamiento al condensador y la de alimentación de vapor a la chaqueta de calentamiento del rehervidor. Se fija la presión del vapor y se registran las temperaturas en el rehervidor, última etapa y el tope de la 
columna (etapa 1). Cuando se alcanza la temperatura de 78-79 $\mathrm{C}$ en el tope de la columna se deja operando a reflujo total y luego se procede a retirar el destilado. En general, la columna durante la etapa de producción opera a ciclos de reflujo total y reflujo mínimo, hasta que se obtenga una baja pureza en el destilado.

Primera corrida.- Para la destilación del sistema etanol-agua se ha usando una carga de calor de $1.29 \mathrm{~kW}$ y un flujo de $2.28 \mathrm{~kg} / \mathrm{h}$ el que se varía con el objeto de mantener la temperatura constante en el rehervidor. La carga inicial es de $1.2 \mathrm{kmol}$ $(23.5$ L) con una composición de $10 \%(\mathrm{v} / \mathrm{v})$.

El perfil de temperatura con los datos experimentales se observan en la Fig. 1.

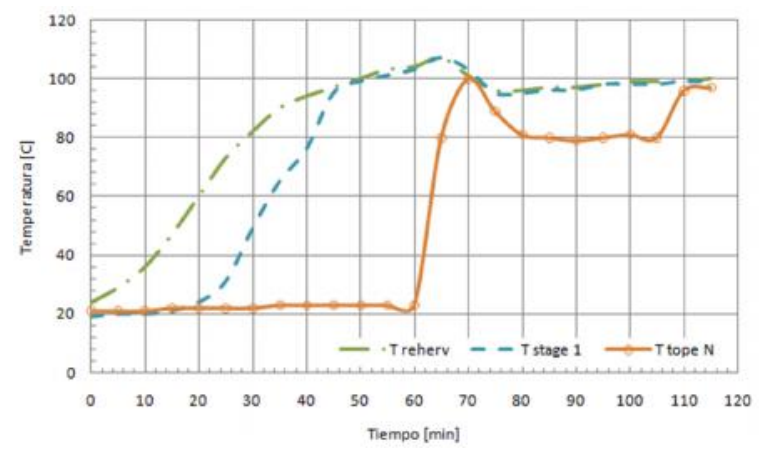

Fig. 1 Perfil de temperatura (primera corrida).

La transferencia de calor en el rehervidor (Treherv) y en la última etapa (Tstage 1) es rápida. El calentamiento del tope de la columna (Ttope $N$ ) se logra después de $60 \mathrm{~min}$ de la apertura de la válvula para admitir el vapor a la chaqueta. Alcanzando rápidamente la temperatura de $79 \mathrm{C}$. La temperatura a la salida del condensador se mantiene en un promedio de $28 \mathrm{C}$, lo que indica que se logra una condensación completa del vapor en el condensador. Se logra separar en el destilado un volumen de $800 \mathrm{~mL}$ con una fracción molar de etanol 40\%(v/v) el primer destilado.

Segunda corrida.- Se destiló una carga inicial de $0.37 \mathrm{kmol}(7.1 \mathrm{~L})$, de una composición $10 \%(\mathrm{v} / \mathrm{v})$ de etanol hasta una carga final de $400 \mathrm{~mL}$. La corrida duró $110 \mathrm{~min}$, la carga de calor usada es de $1.29 \mathrm{~kW}$. Los perfiles de temperatura experimentales se muestran en la Fig. 2. Se puede observar que la temperatura del tope de la columna (Ttope $N$ ) empieza a aumentar después de $20 \mathrm{~min}$ de haber alimentado el vapor a la chaqueta de calentamiento. En este caso no hay una diferencia apreciable en los perfiles de temperatura del rehervidor (Treherv) y de la última etapa (Tstage1).

En esta corrida se obtiene un volumen de $392 \mathrm{~mL}$ de destilado con una fracción molar de $67 \%$ molar el primer destilado.

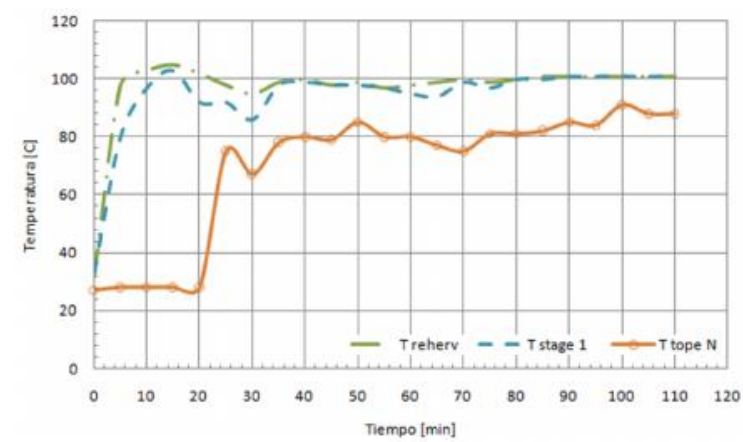

Fig. 2 Perfil de temperaturas (segunda corrida).

\section{SIMULACIÓN}

Los datos usados para la simulación son los obtenidos en dos corridas realizadas en la planta piloto. Según la nomenclatura del simulador los platos se numeran de arriba hacia abajo, incluyendo el condensador y el rehervidor.

De este modo, la etapa 1 corresponde al condensador, la etapa 10 corresponde el plato del fondo de la columna y la etapa 12 al rehervidor.

La simulación del proceso en modo dinámico considera tres etapas:

1. Carga, de mezcla a destilar.

2. Operación a reflujo total, donde se establece el perfil de temperatura y el de retención (holdup) a lo largo de la columna.

3. La obtención del producto

Para el cálculo de propiedades y del equilibrio líquido-vapor se selecciona el modelo termodinámico NRTL y se usa el software de simulación comercial ASPEN Batch Distillation V.7.1.

El estudio de los perfiles de temperatura dentro de la columna es una manera aceptable de validar la simulación y es un criterio aceptado en la industria [4]. Estos perfiles se muestran en la Fig. 3. 


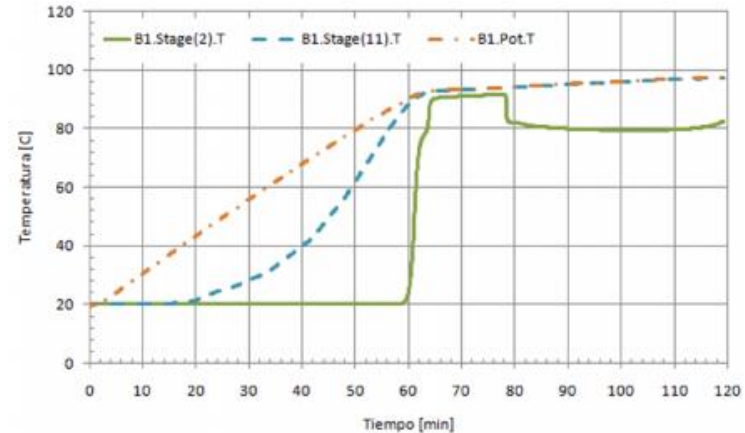

Fig. 3 Perfil de temperatura (Simulación primera corrida).

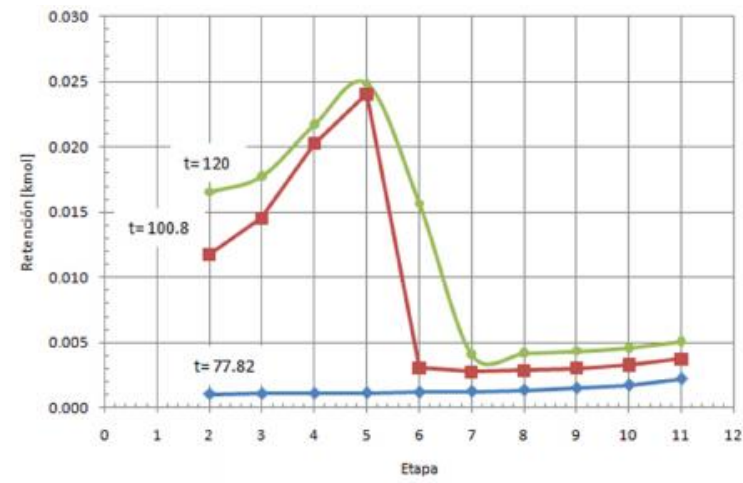

Fig. 4 Retención de líquido a lo largo de la columna (simulación primera corrida).

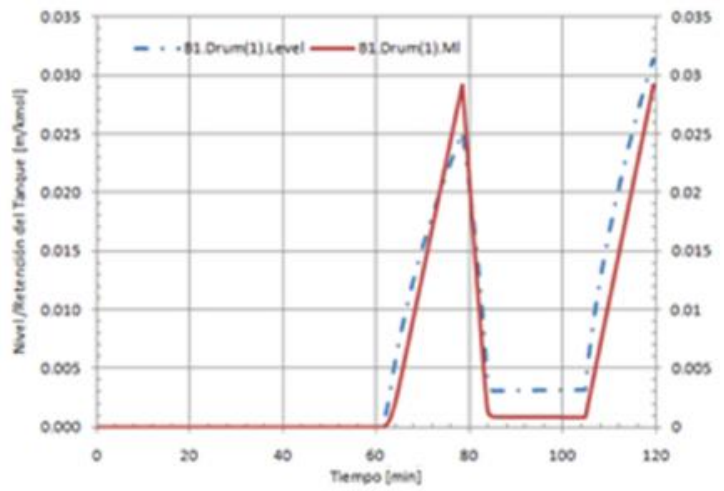

Fig. 5 Comportamiento del tanque de reflujo (primera corrida).

Con los perfiles obtenidos es posible estudiar cual fue el comportamiento de las variables y si son susceptibles de mejorarlas. La

Fig. 4 nos muestra la distribución del líquido retenido en los platos donde se observa que se llega a estado seudo-estacionario a tiempo mayor de $100 \mathrm{~min}$.

La composición del destilado obtenido se observa en la

Fig. 6 logrando alcanzar un 40\% molar de composición para el primer destilado.

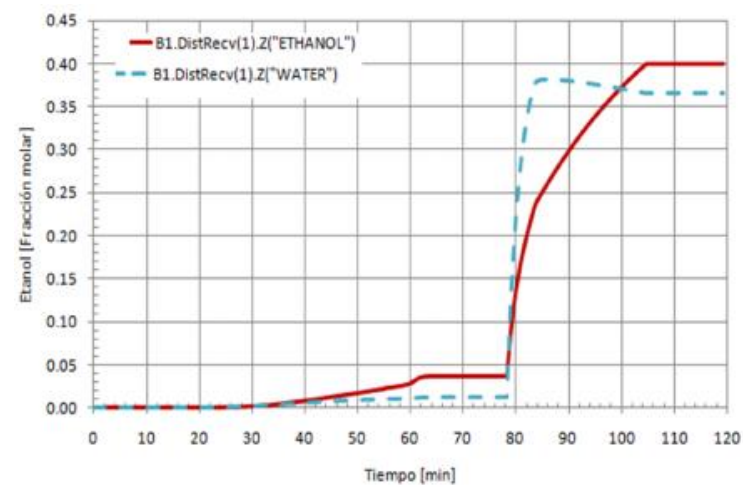

Fig. 6 Composición del destilado (primera corrida).

\section{ANALISIS DE RESULTADOS}

Comparando los perfiles de temperatura se observa que la simulación no describe rigurosamente el proceso de calentamiento del rehervidor. Esto ya que se especificó una carga de calor neto constante. Según las especificaciones del programa, este modo no simula rigurosamente la transferencia de calor y sugiere que existen resistencias que no se han tomado en cuenta en la simulación.

El perfil de temperatura en el plato 1 o tope de la columna es representado satisfactoriamente a lo largo del calentamiento (

Fig. 7, superior).

En el minuto 70 aproximadamente la temperatura aumenta hasta $100 \mathrm{C}$, momento en el cual se varia el flujo de vapor para regular la temperatura a la azeotrópica. Esto no se verifica en el perfil simulado porque la carga de calor neta se consideró constante a lo largo del proceso.

Además, si nos referimos a la hidráulica del plato, la forma del perfil experimental sugiere que la retención en la etapa es mínima mientras que en el simulado es mayor. Cabe indicar que la simulación sugiere considerar una retención la cual, para nuestro caso, se ha fijado en referencia a 
la pureza del destilado obtenida experimentalmente.
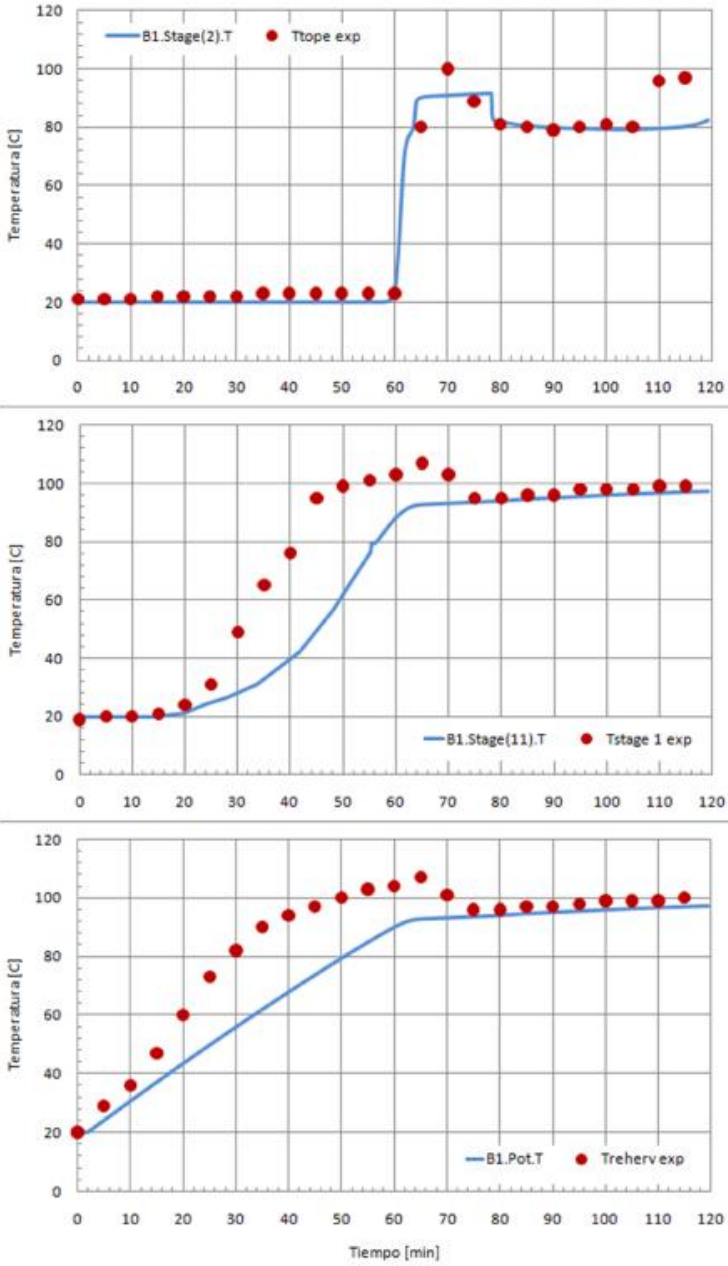

Fig. 7 Comparación de resultados simulados con los experimentales de los perfiles de temperatura (primera corrida).

De acuerdo a la simulación se logra obtener una pureza de aproximadamente $74 \%$ molar en el condensador, manteniendo un nivel de $0.026 \mathrm{~m}$ en el tanque de reflujo. Sin embargo, al extraer destilado provoca una disminución en la retención del tanque de reflujo que desestabiliza el sistema y se obtiene una menor composición en el destilado (

Fig. 5).

Cabe resaltar que la cantidad de carga inicial en la segundo corrida es pequeña de modo que es posible que no se haya alcanzado una retención adecuada para alcanzar la pureza deseada. Sin la retención adecuada el sistema se desestabiliza fácilmente frente a cualquier perturbación (e.g., extracción de destilado).

No se ha logrado simular satisfactoriamente la etapa de calentamiento en el rehervidor y la etapa 10, lo cual se debe a la manipulación continua de la válvula de ingreso de vapor al rehervidor (

Fig. 7, medio e inferior).
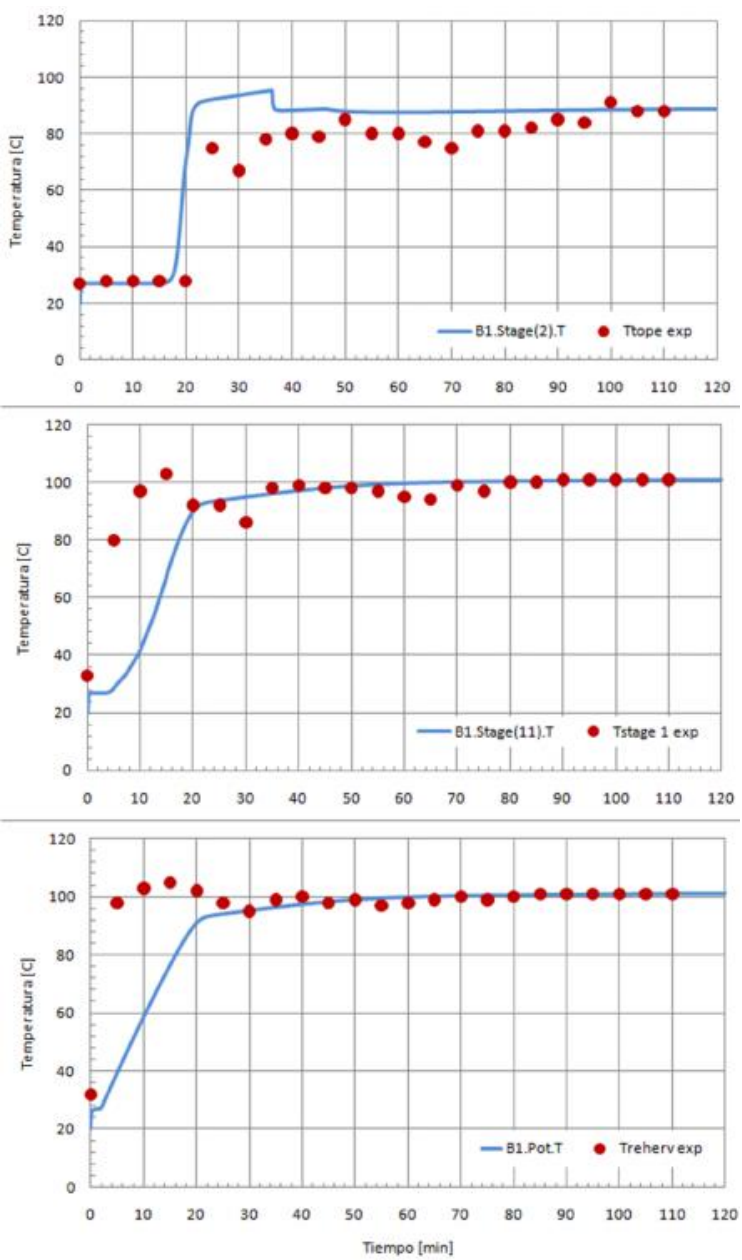

Fig. 8 Comparación de resultados simulados con los experimentales de los perfiles de temperatura (segunda corrida).

La simulación para la segunda corrida es satisfactoria para la etapa a reflujo total para el rehervidor y la etapa 10. Sin embargo, no se ha logrado mantener la temperatura en el tope de la columna aun cuando el reflujo tienda a infinito (Fig. 8).

Para automatizar la planta piloto se considera en la simulación dos controladores. Uno que controla la composición en el condensador manipulando la 
relación de reflujo y otro que controla la temperatura en el tope de la columna dentro del rango de 79-78 $\mathrm{C}$, manipulando la carga de energía al rehervidor. El simulador tiene definido un controlador que controla el nivel del tanque de reflujo, el cual se consideró dentro de la estrategia de control.

Para mejorar el proceso se considera reducir el tiempo de calentamiento sin afectar la composición del destilado. De acuerdo a los estudios realizados mientras más rápido es el calentamiento la máxima composición del etanol que se puede obtener disminuye, es por ello que se establece un rango de variación de $0-0.01 \mathrm{GJ} / \mathrm{h}$ para la variable manipulada.

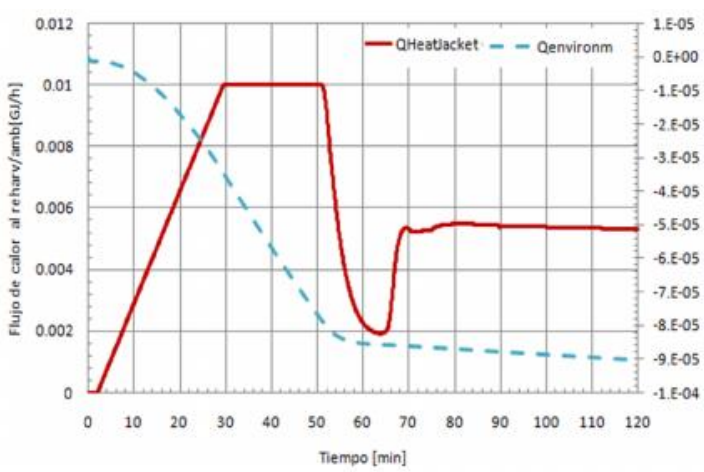

Fig. 9 Flujo de calor de la chaqueta de calentamiento al rehervidor y al medio ambiente.

Con este análisis para mejorar los resultados experimentales podemos sugerir operar llevando a cabo las siguientes acciones. Cargar la mezcla. En la etapa de calentamiento a reflujo total el controlador de nivel y el de reflujo se fijan en modo manual, dejando actuar el controlador de temperatura del tope de la columna, de esta manera se logra que los perfiles de temperatura y de retención se establezcan en los platos de la columna y el tanque de reflujo.

El comportamiento del calor se muestra en la Fig. 9. Como se puede observar el flujo de calor alcanza un valor mínimo de $0.002 \mathrm{GJ} / \mathrm{h}$ cuando la temperatura en el tope de la columna llega 78.2 C (

Fig. 10, B1. Stage(2).T).
Luego se cambia el modo de operación de los controladores de manual a automático, dejándolos actuar por un tiempo (30 min) de esta manera se consigue que todas las variables lleguen al estado estacionario (e.g., reflujo, nivel, temperaturas, composición). Luego se puede obtener el destilado.

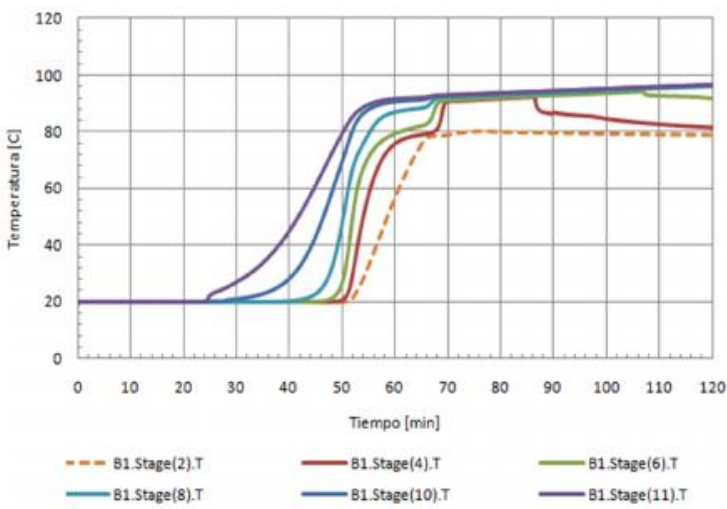

Fig. 10 Perfiles de temperatura en función del tiempo en los platos de la columna.

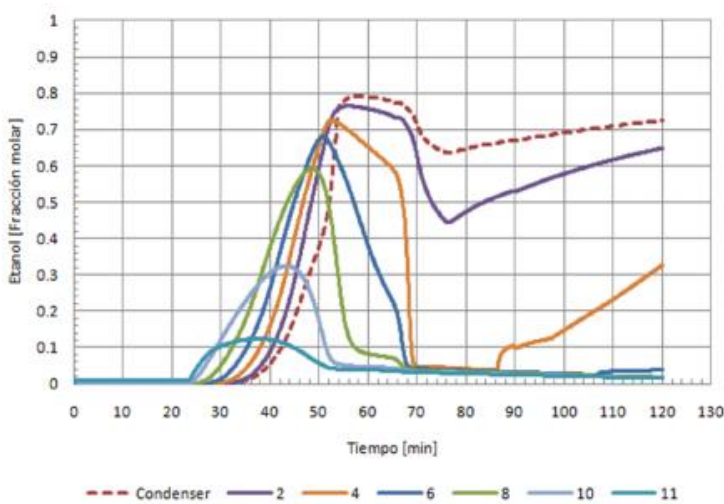

Fig. 11 Perfiles de composición en función del tiempo en los platos de la columna.

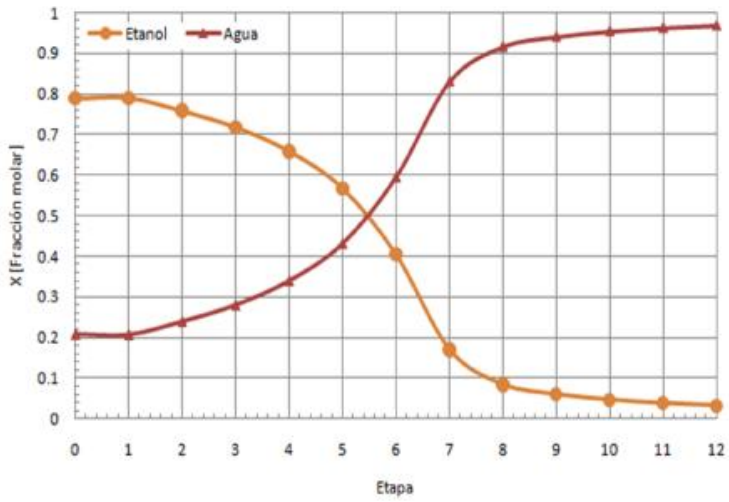


Fig. 12 Distribución de etanol y agua a lo largo de la columna, en el estado estacionario y máxima recuperación.

Cuando el flujo de destilado aumenta la composición empieza a disminuir (

Fig. 11), por lo cual el flujo debe ser tal que no desestabilice el sistema vaciando el tanque de reflujo. De acuerdo a los perfiles, si bien la temperatura en el tope no varía, las composiciones caen rápidamente y el sistema requiere más de 40 min en volver al estado estacionario. Lo que indica que para una segunda obtención de destilado se debe esperar un tiempo mayor.

La distribución de los componentes muestran que existe una buena distribución a lo largo de las etapas de separación, ocurriendo el punto de inflexión en aproximadamente la mitad de la columna (Fig. 12).

En general, con esta configuración se ha logrado obtener una composición del 78\% molar de etanol. $\mathrm{Si}$ al extraer destilado solo lo hacemos hasta que la composición disminuya hasta el $67 \%$, en promedio la composición del destilado será del 73\% molar. Mejorando de esta manera la composición obtenido en las pruebas experimentales.

\section{CONCLUSIONES}

Los perfiles de temperatura simulados coinciden satisfactoriamente con los perfiles experimentales en la etapa productiva.

La etapa de calentamiento no se ha logrado simular rigurosamente, ya que no se tiene información exacta referente al rehervidor para considerar todas las resistencias del mismo.

Importante información sobre la hidrodinámica del plato se ha logrado establecer, denotando la poca retención que ocurre en las etapas.

Se ha logrado establecer un protocolo e trabajo para mejorar la separación del etanol.

Es necesario implementar controladores lo suficientemente rápidos para manipular los flujos de reflujo y destilado, de modo que la obtención del producto no se haga a expensas de disminuir la retención en el tanque de reflujo.

El simulador ASPEN Batch Distillation V7.1 es capaz de simular la inercia termal de la planta debido a que en la etapa de carga la planta está a temperatura ambiente.

El simulador considera automáticamente la existencia de un controlador de nivel que manipula la salida de destilado, lo cual condiciona la configuración de control que se puedan evaluar. En general, se ha logrado simular en forma realista y el simulador demuestra ser una buena herramienta para analizar, mejorar y optimizar el proceso de destilación batch.

\section{REFERENCIAS}

1. Jr., Costa, E. F., Vieira, R. C. et al., "Dynamic Simulation of High-Index Models of Batch Distillation Processes." Latin American Applied Research 33, pp. 155-160, 2003.

2. Arndt, Mehlhorn., A., Nougués, J. M., Puigjaner, L., "Using rate-based approach under consideration of different contacting regimes for multicomponent", Computers \& Chemical Engineering, 22, Sup. 1, pp. S645S648, 1998.

3. Bonsfills, A., Puigjaner, L., "Batch distillation: Simulation and Experimental Validation", Chemical Engineering and Processing 43, pp. 1239-1252, 2004.

4. Wang, L., P. Li, et al, "A startup model for simulation of batch distillation starting from a cold state", Computers \& Chemical Engineering 27(10), pp. 1485-1497, (2003)

5. Schneider, R., C. Noeres, et al., "Dynamic modeling and simulation of reactive batch distillation", Computers \& Chemical Engineering_25(1), pp. 169-176, (2001)

6. Fang, J., Li, C., et al., "A Quasi-steady-state Model for Numerical Simulation of Batch Extractive Distillation", Chinese Journal of Chemical Engineering 18(1), pp. 43-47, (2010).

Correspondencia: elianejm@hotmail.com 